\title{
Human Transcription Factor YY1 Represses Human Immunodeficiency Virus Type 1 Transcription and Virion Production
}

\author{
DAVID M. MARGOLIS, ${ }^{*}$ MOHAN SOMASUNDARAN, AND MICHAEL R. GREEN \\ Program in Molecular Medicine, University of Massachusetts Medical Center, Worcester, Massachusetts 01605
}

Received 9 September 1993/Accepted 27 October 1993

\begin{abstract}
The transcriptional activity of human immunodeficiency virus type 1 (HIV-1) is affected by many cellular factors. Homologies near the HIV-1 initiator region to the DNA-binding sequences of YY1, a multifunctional transcription factor known to regulate diverse viral and cellular promoters, suggested that YY1 might regulate HIV-1. Antibody to YY1 blocked the formation of complexes by HeLa cell nuclear extract and a DNA oligonucleotide encoding the HIV-1 initiator region. HIV-1 long terminal repeat (LTR) expression, as measured the expression of a transfected LTR-CAT reporter gene, was repressed more than 12-fold by the cotransfection of a YY1 expression vector. HIV-1 production by both COS-1 and CEM cells after transfection of an infectious molecular HIV-1 clone was repressed 7- to 20 -fold by cotransfection of a YY1 expression vector. HIV-1 production was also decreased threefold in a CD4-positive lymphocyte cell line chronically infected with HIV-1 (8E5) after transfection of YY1. In situ hybridization studies confirmed that YY1 reduced HIV-1 RNA expression. YY1 may play an important role in the regulation of HIV-1 LTR expression in vivo and virus production by infected cells.
\end{abstract}

Intensive study has shown that the long terminal repeat (LTR), the promoter of human immunodeficiency virus type 1 (HIV-1), responds to numerous cellular factors $(10-14,22,31)$. While many of these factors augment LTR expression, no cellular factor has been shown to directly decrease production of HIV-1 virions by infected cells. Nevertheless, HIV-1 mRNA is expressed in only a small percentage of infected cells $(6,7$, 25). The HIV-1 activating protein Tat powerfully stimulates transcription of all viral messages (14). Factors that regulate LTR expression in the basal state, before the accumulation of a threshold quantity of Tat, must play a central role in the restriction of HIV-1 mRNA expression.

Factors known to bind to and regulate the HIV-1 LTR are found in abundance in lymphocytes, a primary cellular target of HIV-1. YY1, a multifunctional human transcription factor, regulates both viral and lymphocyte promoters (3, 8, 26-28). USF recognizes the HIV-1 initiator region and shares with YY1 an affinity for the TdT initiator $(5,27)$. Homologies between DNA sequences known to bind YY1 and the HIV-1 initiator region suggested that YY1 might regulate the HIV-1 promoter.

We report evidence that YY1 recognizes the initiator region of the HIV-1 LTR. YY1 decreased LTR-directed gene expression. Cotransfection of a YY1 expression vector with an infectious molecular HIV-1 clone resulted in diminished virus production in several cell lines. Similarly, expression of YY1 in chronically HIV-1-infected T-cell lines reduced the expression of HIV-1 RNA and viral particles. As YY1 did not significantly repress Tat-activated LTR expression but repressed HIV-1 production in vivo, we suggest that YY1 exerts its effect at a critical stage in the HIV-1 life cycle.

\footnotetext{
* Corresponding author. Mailing address: Program in Molecular Medicine, University of Massachusetts Medical Center, 373 Plantation St., Suite 309, Worcester, MA 01605. Phone: (508) 856-1987. Fax: (508) 856-5473.
}

\section{MATERIALS AND METHODS}

Plasmids and cell lines. The T-cell lines CEM, 8E5, and $\mathrm{ACH} 2$ were maintained in suspension culture in RPMI 1640 medium (ICN Biochemicals, Costa Mesa, Calif.) supplemented with $10 \%$ fetal bovine serum (GIBCO, Grand Island, N.Y.). COS-1 and HeLa cells were grown in monolayers in Dulbecco's modified Eagle's medium (GIBCO) supplemented with $10 \%$ fetal bovine serum. The HIV-1 LTR-chloramphenicol acetyltransferase (CAT) reporter gene construct pILIC (1), the infectious molecular clone of HIV-1 pNL4-3 (1), the Tat expression vector pcTat (30), and the $\beta$-galactosidase expression plasmid pSV- $\beta$ gal (19) were prepared by the standard alkaline lysis method. pCMV-YY1 was constructed by insertion of a StyI-EcoRI fragment of the YY1 cDNA plasmid (28) into the Bam HI site of the expression vector pCMV-bam/ neo3 (15).

Transfections, CAT assays, and p24 ELISA. Monolayer cells were transfected with $20 \mu \mathrm{g}$ of plasmid DNA by the calcium phosphate procedure (21) with $5 \mu \mathrm{g}$ of LTR-CAT reporter, 5 or $10 \mu \mathrm{g}$ of effector DNA, and carrier DNA. Suspension cultures were transfected by the DEAE-dextran method (21) with $1.5 \mu \mathrm{g}$ of LTR-CAT reporter or $2 \mu \mathrm{g}$ of pNL4-3 and 1 to $4 \mu \mathrm{g}$ of effector DNA. CAT assays were performed $48 \mathrm{~h}$ after normalization for total protein content (21). To measure transfection efficiency, pSV- $\beta$ gal was cotransfected and $\beta$-galactosidase assays were performed as described previously (19). Aliquots of culture medium were sampled for detection of HIV-1 p2 $4^{\text {gag }}$ protein. Assays were performed with an antigen capture Coulter enzyme-linked immunosorbent assay (ELISA) kit according to the manufacturer's instructions.

Antibody depletion in EMSAs. Electrophoretic mobility shift assays (EMSAs) with HeLa nuclear extract were performed as described previously with an oligonucleotide corresponding to the -16 to +27 sequence of the HIV-1 LTR $\left(5^{\prime}\right.$-TGCTTTTT GCCTGTACTGGGTCTCTCTGGTTAGACCAGATCTGA G-3' [21]). The EMSA with YY1 protein was performed as described by Shi et al. (28). For depletion experiments, YY1 antiserum or preimmune serum (gift of T. Shenk) was added to 


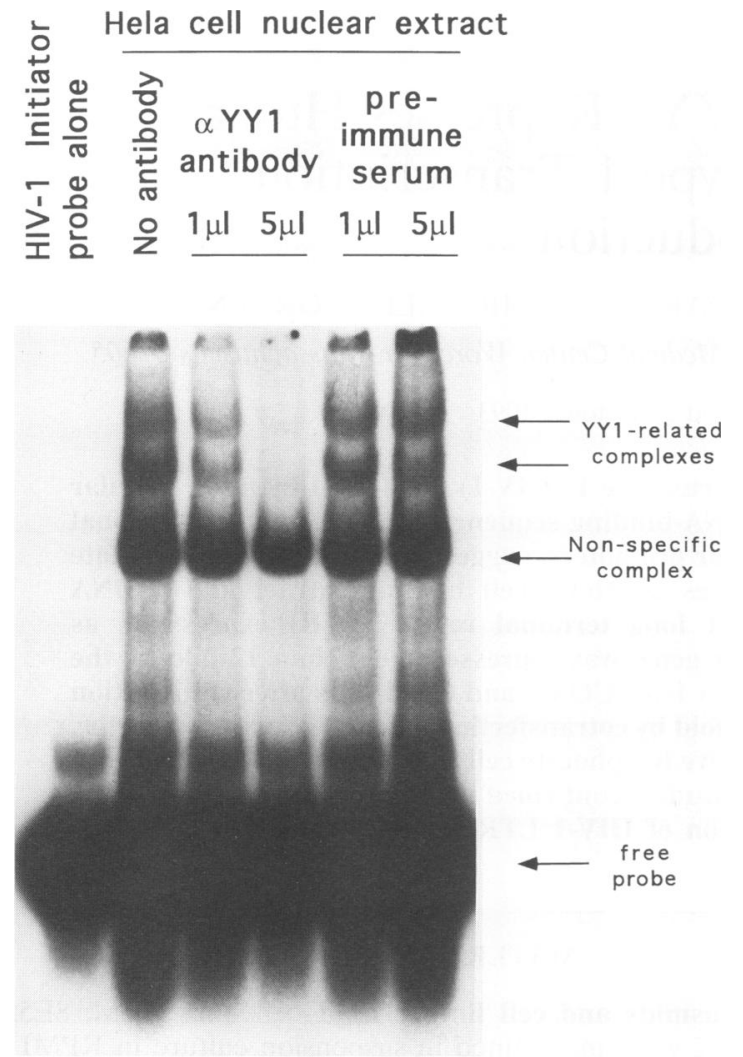

FIG. 1. Electrophoretic mobility shift complexes formed by HeLa cell nuclear extract and an oligonucleotide encoding the HIV-1 LTR from -17 to +25 with respect to the transcriptional start. Formation of complexes previously shown to be specifically inhibited by unlabeled oligonucleotide (20) was inhibited by YY1 antiserum but not by preimmune serum. A nonspecific complex (20) was unaffected by YY1 antiserum or preimmune serum.

the EMSA reaction mixture and incubated for a further hour at $4^{\circ} \mathrm{C} ; 5 \mu \mathrm{l}$ of $10 \%$ Staphylococcus aureus Cowan cells (Pansorbin; Calbiochem, La Jolla, Calif.) was then added and incubated for an hour at $4^{\circ} \mathrm{C}$. The reaction mixture was centrifuged, and the supernatant was run on an acrylamide gel as described before (21).

Detection of HIV-1 RNA by in situ hybridization. Cells were fixed in $4 \%$ paraformaldehyde, and in situ hybridization with alkaline phosphatase detection was performed as described before (29).

\section{RESULTS}

YY1 antibody interferes with a DNA-protein interaction near the HIV-1 initiator region. Several lines of evidence suggest that YY1 can recognize the HIV-1 LTR initiator region. Bacterially synthesized YY1 protein (gift of Y. Shi) bound an oligonucleotide encoding the LTR -16 to +27 region in EMSAs (data not shown). YY1 is present in HeLa cell nuclear extracts (28). In EMSA competition experiments with HeLa nuclear extract, the labeled LTR $-16 /+27$ probe, and competitor representing the $\kappa E 3^{\prime}$ YY1 binding site (8) of the immunoglobulin kappa light chain $3^{\prime}$ enhancer $\left(5^{\prime}\right.$-CCAC CTCCATCTTG- $3^{\prime}$ ), more than a 30 -fold molar excess of $\kappa E 3^{\prime}$ competitor was required to prevent complex formation. In the converse experiment, complexes bound to the labeled $\kappa \mathrm{E} 3^{\prime}$

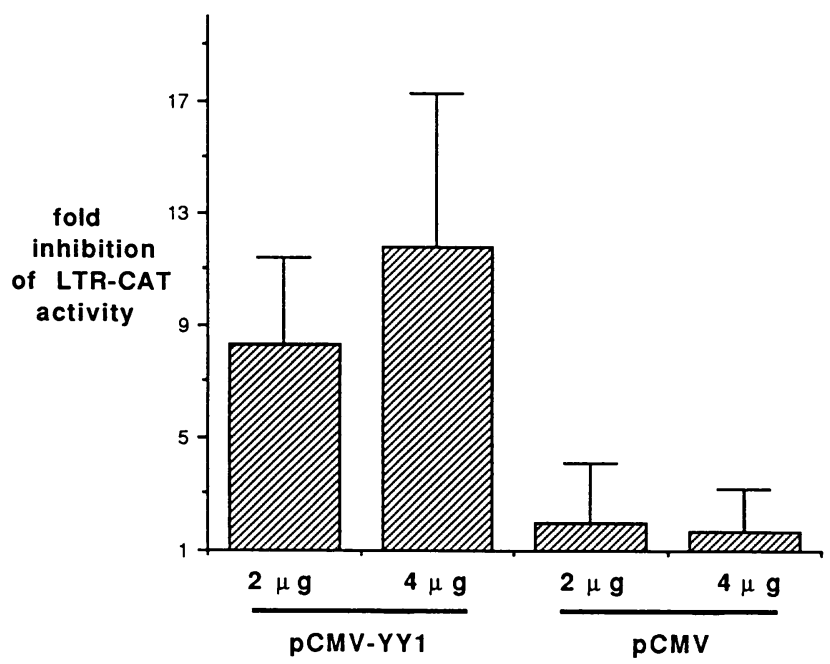

FIG. 2. Inhibition of LTR-directed CAT expression by YY1 in transfected CEM cells. Fold inhibition was calculated by dividing the percentage of acetylated chloramphenicol when the HIV-1 LTR-CAT reporter was transfected alone by the percentage of acetylated chloramphenicol when the reporter was cotransfected with pCMV or pCMV-YY1. Values of less than 1 indicate activation; cotransfection of pCMV resulted in weak activation or inhibition, and cotransfection of PCMV-YY1 resulted in significant inhibition. Values represent the mean and standard deviation for four experiments.

probe were preferentially inhibited by a 10 -fold molar excess of the LTR oligonucleotide (data not shown).

The effect of YY1 antiserum on EMSA complexes formed with the LTR oligonucleotide provided further evidence that YY1 is involved in complex formation. In previous EMSA studies, we have found one nonspecific and several specific complexes formed by this oligonucleotide and HeLa nuclear extract (21). YY1 antiserum interfered with the formation of specific complexes by a HeLa nuclear protein and the LTR initiator region, while preimmune serum did not interfere with complex formation (Fig. 1). Nonspecific complexes were unaffected by YY1 antiserum.

YY1 is capable of direct interaction with both DNA and other cellular factors (19). Together, our findings suggest that YY1 or an immunologically related protein complex recognizes the HIV-1 initiator region.

YY1 represses LTR-directed CAT activity. The ability of YY1 to decrease LTR-directed expression of CAT was tested. CEM cells, a CD4-positive lymphocyte line, were cotransfected with the HIV-1 LTR-CAT reporter and pCMV-YY1. In control experiments, the reporter was cotransfected with the unmodified expression vector pCMV-bam/neo3. Compared with the activity of the reporter transfected with the unmodified vector, pCMV-YY1 caused up to 12-fold repression in a dose-dependent manner (Fig. 2). Expression of pSV-ßgal, as measured by assays of $\beta$-galactosidase activity, varied by less than $10 \%$ between selected cultures. Repression was not dependent on promoter sequences upstream of the NF- $\mathrm{B}$ sites, as a reporter (pLTR $\mathrm{dl}^{-525 /^{-}} 98$ [24]) lacking those sequences was repressed to the same extent as the construct carrying the complete LTR (data not shown). YY1 had a marginal effect on activation mediated by cotransfection with pcTat (data not shown).

YY1 decreases HIV-1 production in nonlymphoid cells. To assess the biological relevance of the repression mediated by 
A

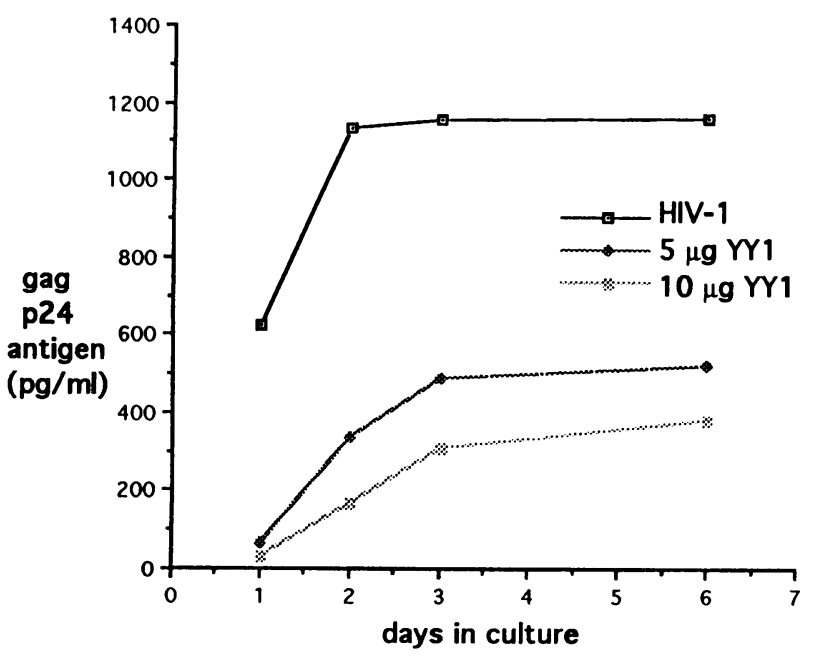

B

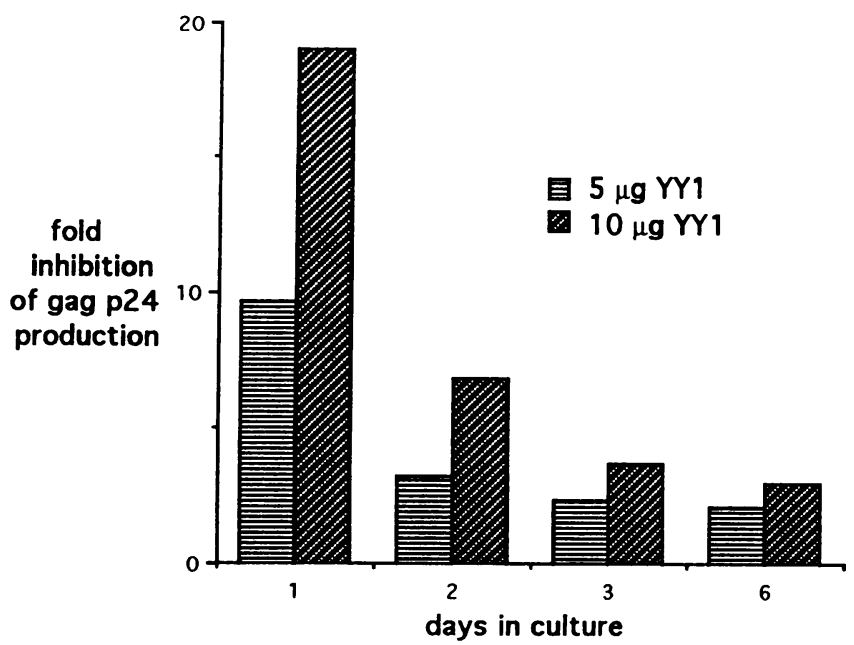

FIG. 3. Inhibition of $\mathrm{p} 24^{\text {gag }}$ production in COS-1 cells. HIV-1 DNA was transfected with pCMV or pCMV-YY1. (A) Concentrations of p24 measured after transfection. For simplicity, data are shown only for $10 \mu \mathrm{g}$ of pCMV (designated HIV-1); the values for $5 \mu \mathrm{g}$ of pCMV were nearly identical. Results are representative of three experiments. (B) Fold inhibition was calculated by dividing p24 values measured after cotransfection of HIV-1 DNA and pCMV by values measured after cotransfection of HIV-1 and the indicated amount of pCMV-YY1.
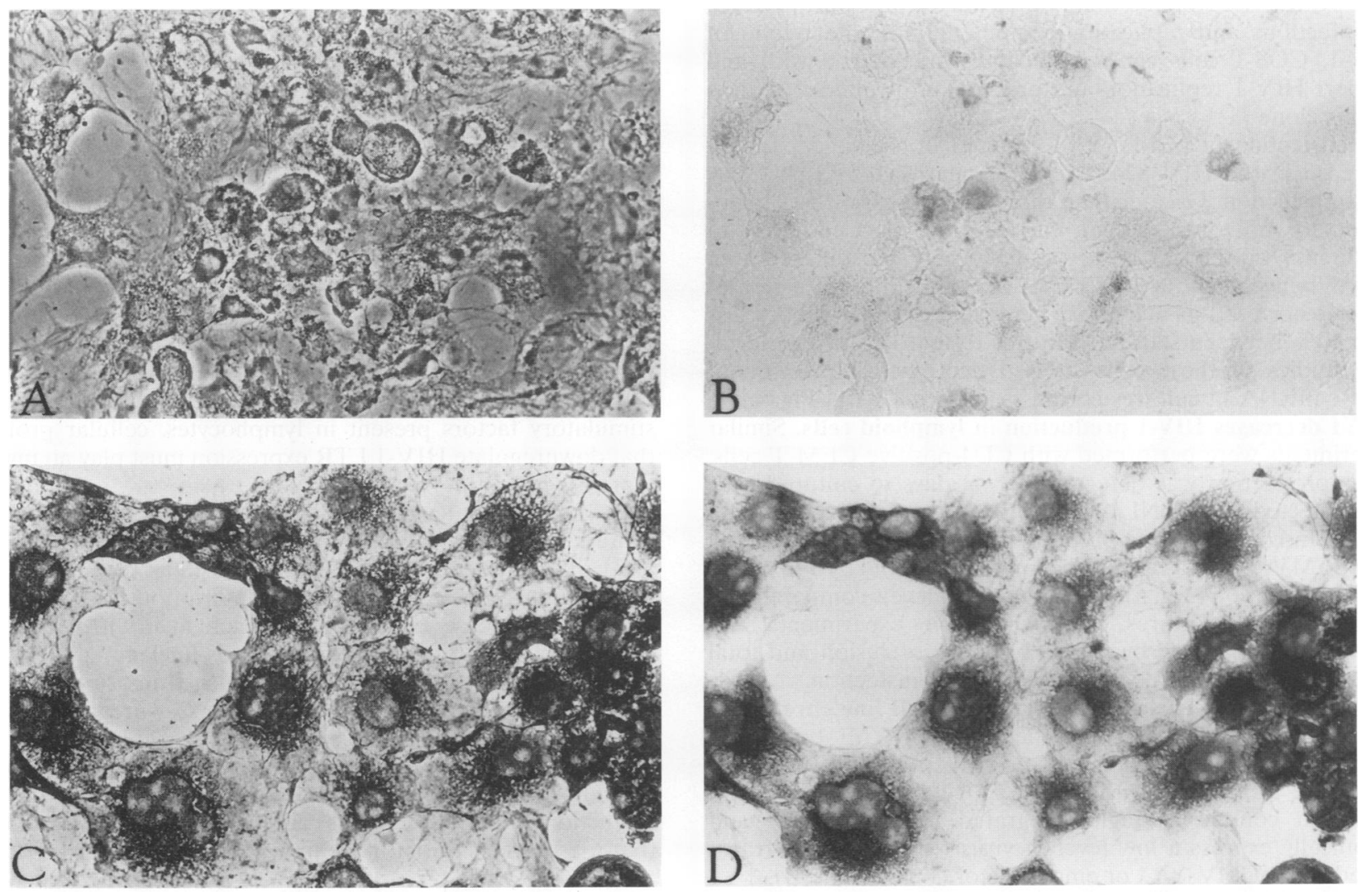

FIG. 4. One day after transfection of COS-1 cells, HIV-1 mRNA was detected by in situ hybridization with alkaline phosphatase (27). Nick-translated, biotinylated whole HIV-1 genomes were used as probes. Cells transfected with HIV-1 and pCMV-YY1 under phase (A) and bright light (B); little dark hybridization signal is seen. Control cells transfected with HIV-1 and unmodified CMV expression vector under phase (C) and bright light (D); copious dark hybridization signal is seen. 
A

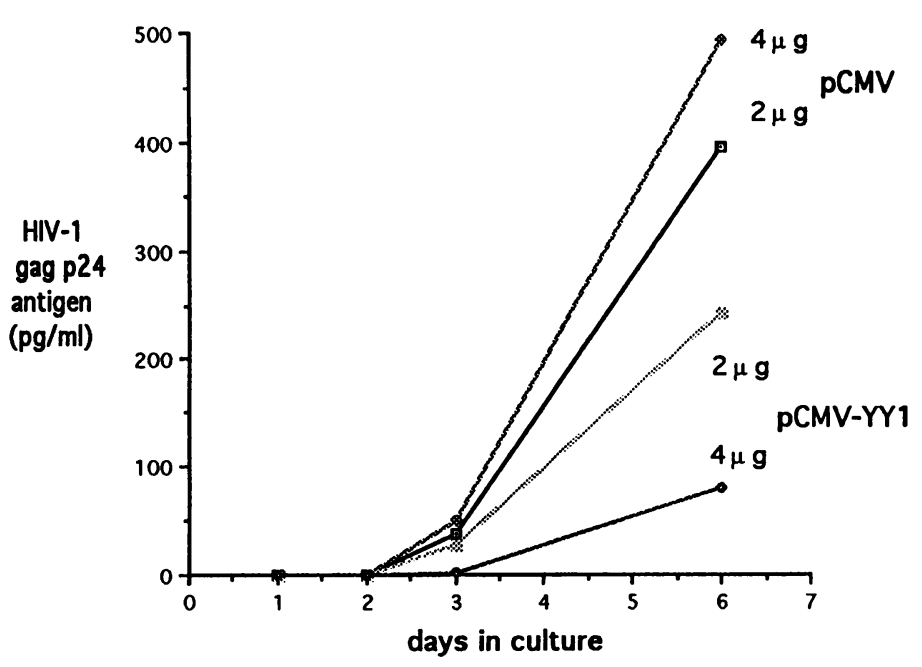

B

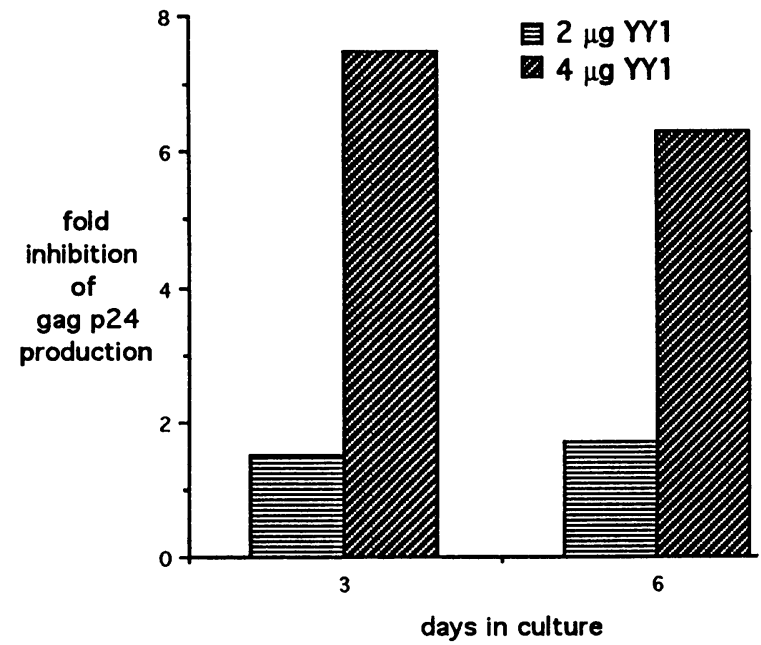

FIG. 5. Inhibition of p2 $4^{\text {gag }}$ production in CEM cells. Experiments and data calculations were performed as for Fig. 3. (A) Concentration of p24. Results are representative of three experiments. (B) Fold inhibition.

YY1, the effect of YY1 on the expression of virus in a model of acute infection was studied. COS-1 cells were cotransfected with pCMV-YY1 or the unmodified vector and with pNL4-3, an infectious and replication-competent molecular clone of HIV-1. COS-1 cells can be efficiently transfected with and support HIV-1 replication and production of infectious virus by this clone (1).

Transfection of pCMV-YY1 resulted in a dose-dependent decrease in the HIV p24gag level of nearly 20 -fold by 1 day posttransfection. Greater than threefold repression was maintained for up to 10 days in culture (Fig. 3). Cells grew to confluency by day 3 ; there was no observable difference in growth between cultures. YY1 did not affect the expression of a cotransfected $\beta$-galactosidase gene; expression varied by less than $10 \%$ between selected cultures tested. In situ hybridization studies of these cells showed decreased expression of HIV-1 mRNA in cultures cotransfected with YY1 (Fig. 4).

YY1 decreases HIV-1 production in lymphoid cells. Similar experiments were performed with CD4-positive CEM T cells. Virus production was detected after 3 days in cultured cells cotransfected with pNL4-3 and the unmodified expression vector. In contrast, virus production in cultures cotransfected with pCMV-YY1 was diminished up to sevenfold by 3 to 6 days after transfection (Fig. 5) and then rose to levels comparable to those in other cultures. Throughout the experiments, cell viability, as measured by trypan blue dye exclusion and total cell number, was not decreased by YY1 transfection.

YY1 decreases virus production in a T-cell line chronically infected with HIV-1. To test the effect of YY1 on HIV-1 expression in cells which constitutively express the virus, 8E5 cells were transfected with YY1. 8E5 cells are CEM-derived cells that contain a single integrated HIV-1 genome and chronically express a low level of virus (9). Cells were transfected with pCMV-YY1 or empty vector (Fig. 6A and B). YY1 repressed HIV-1 production up to threefold 2 days after transfection (Fig. 6C). In situ studies (not shown) again showed decreased HIV-1 mRNA expression in cells cotransfected with YY1.
$\mathrm{ACH} 2$ cells are similar to $8 \mathrm{E} 5$ cells but produce high titers of HIV-1 upon stimulation by tumor necrosis factor alpha or tetradecanoyl phorbol acetate (TPA) (4). Expression of virus in the basal state, before TPA stimulation, was repressed by transfection of pCMV-YY1. However, YY1 did not block the induction of virus after TPA stimulation (data not shown).

\section{DISCUSSION}

Integrated HIV-1 DNA is now known to be present in a significant number of CD4-positive lymphocytes during all stages of HIV-1 infection. Viral mRNA, however, is expressed in only a fraction of these cells $(6,7,25)$. Many cellular factors have been shown to increase HIV-1 mRNA expression (10-14, $22,31)$. Initial reports that the HIV-1 nef gene can downregulate viral expression were refuted by later studies $(17,20)$. As mRNA expression is restricted despite the abundance of stimulatory factors present in lymphocytes, cellular proteins that downregulate HIV-1 LTR expression must play an important role in the life cycle of HIV-1.

In vitro studies have shown that the cellular factor USF can repress transcription via a binding site in the $\mathrm{U} 3$ region (11) and can activate transcription through a site at the initiator (5). LBP-1, first reported to activate transcription (13), has been shown to decrease HIV-1 transcription in in vitro reactions (16). The in vivo role of these factors is unclear.

We have shown that YY1, a cellular transcription factor thought to be widely distributed (28), can downregulate HIV-1 expression. Evidence suggests that YY1 recognizes a site near the initiator region and represses HIV-1 transcription. It should be emphasized that we present no direct evidence that a functional initiator element exists in the HIV-1 LTR (23) or that YY1 directs initiator-mediated transcription of the LTR, as it can in other promoters (27). It is interesting that when acting through bona fide initiator elements, YY1 activates transcription $(2,27)$. Our findings support the hypothesis that the effect exerted by YY1 is critically dependent on interactions with cellular factors. 

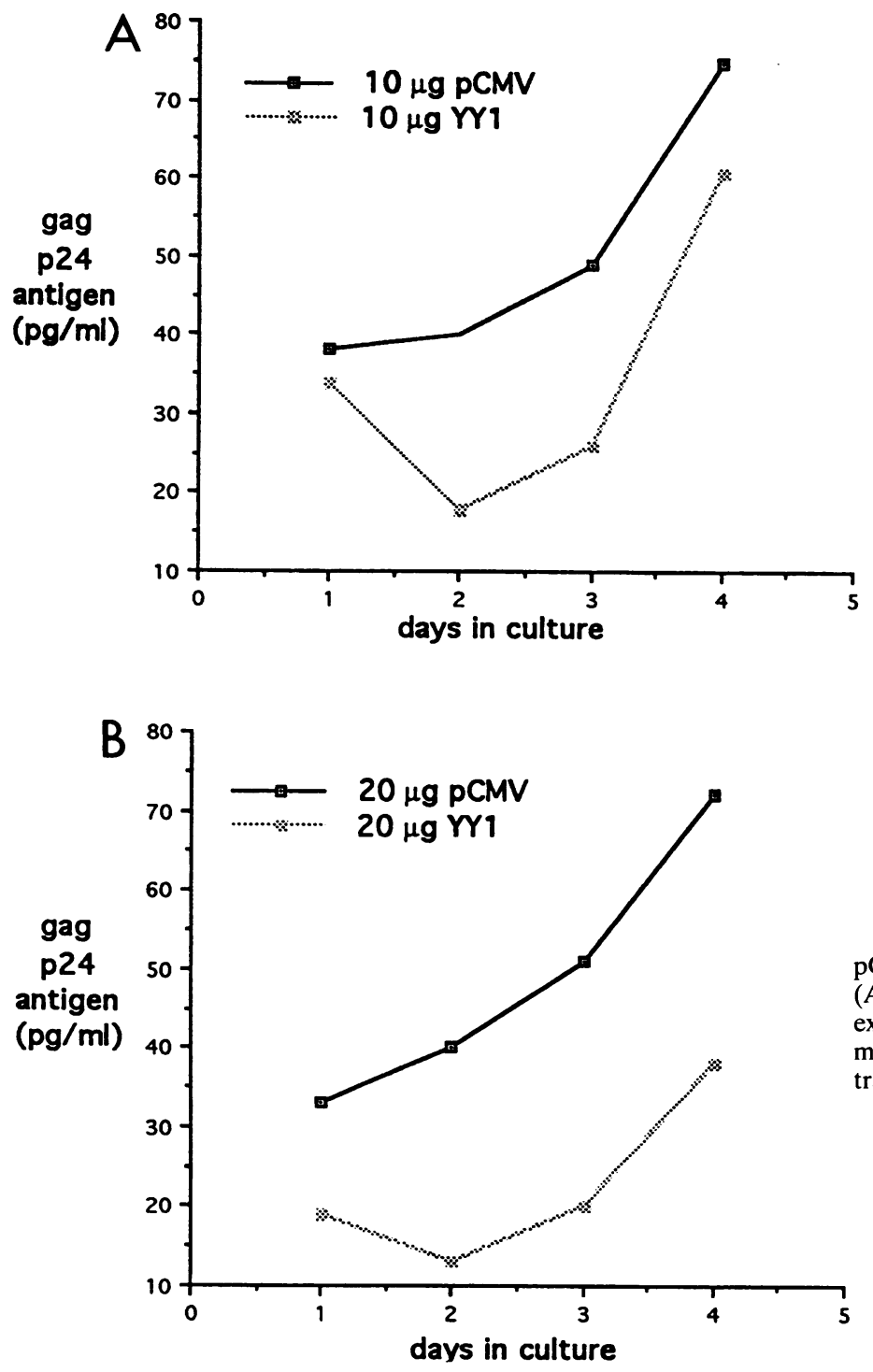

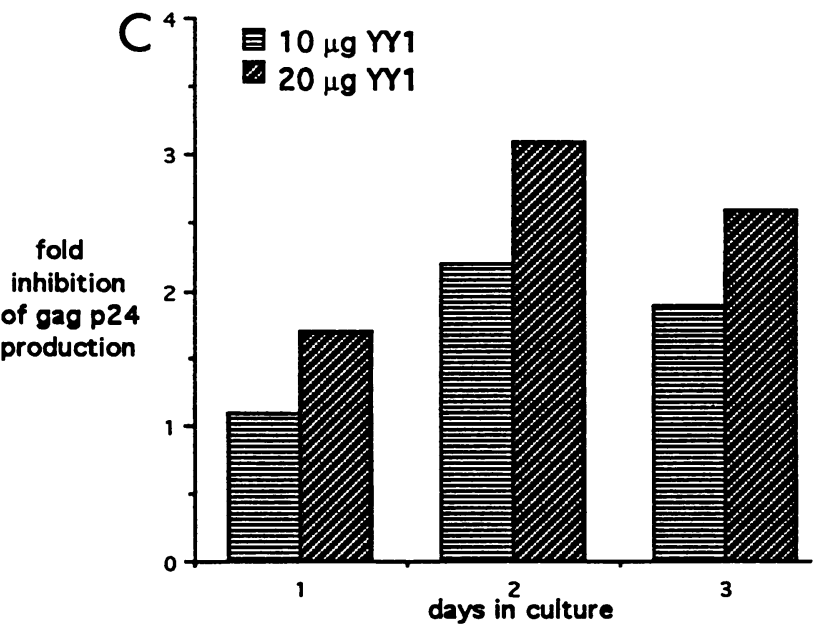

FIG. 6. Inhibition of $\mathrm{p} 24^{\mathrm{gag} g}$ production in $8 \mathrm{E} 5$ cells. Only pCMV or pCMV-YY1 was transfected, as 8E5 cells chronically produce HIV-1. (A and B) Concentration of p24. Results are representative of three experiments. (C) Fold inhibition was calculated by dividing p24 values measured after transfection with pCMV by values measured after transfection of the indicated amount of PCMV-YY1.
Repression was not accounted for by cell death induced by transfection of YY1, as viability measured by trypan blue dye exclusion was unaffected by YY1. $\beta$-Galactosidase expression was unaffected by YY1, suggesting that YY1 does not cause nonspecific repression of transcription. In situ hybridization studies demonstrate that repression is mediated at the level of transcription.

For the first time, in tissue culture models of both acute and chronic infection, we have shown that a cellular transcription factor can decrease HIV-1 production. We observed repression in both nonlymphoid (COS-1) and lymphoid (CEM) cells.

Host cell differences may explain the different kinetics of repression observed in these experiments. COS-1 cells are CD4 negative, and so superinfection of cells by progeny virions could not occur. It has been suggested that, after a transient period of virus production, transfected HIV-1 DNA fails to stably integrate into the chromosome of nonlymphoid cells (1). These factors account for the plateau of virus production and sustained effect of YY1 (see Fig. 3) in COS-1 cells. CEM CD4-positive lymphocytes are susceptible to continued rounds of viral replication and reinfection. Consistent with the tran- sient nature of YY1 expression, the peak effect of YY1 was seen 2 to 3 days after transfection of CEM cells. Similarly, maximal repression of virus production in $8 \mathrm{E} 5$ cells, a line that chronically produces HIV-1, coincided with the expected peak of YY1 expression.

Repression by YY1 was not irreversible. Repression in $\mathrm{ACH} 2$ cells could be ablated by mitogen activation. In the context of transfection of a tat expression vector, YY1 is not capable of significant repression of Tat-activated transcription of an LTR-CAT reporter. However, YY1 can repress HIV-1 expression in models of acute and chronic infection in which the amount of Tat present may be more biologically relevant.

YY1 can form protein-protein contacts with SP1 and perhaps other basic transcription factors (18). Repression by YY1 is likely to be mediated by such interactions. It will be important to determine whether YY1 interacts directly with the LTR or in cooperation with other factors and whether YY1 competes with other factors that can recognize the HIV-1 initiator region.

Our observations suggest that YY1 restricts HIV-1 transcription and production in the unstimulated state but allows 
viral expression after appropriate stimulation. The majority of infected cells probably exist in such a state of "transcriptional latency," capable of virus production upon appropriate activation. It will be important to determine the relative levels of YY1 present in a variety of cell types under a variety of physiological conditions. Understanding of the mechanism of YY1 action and how repression by YY1 is overcome by activating signals may yield important insights into this critical step in the life cycle of HIV-1.

\section{ACKNOWLEDGMENTS}

We thank Y. Shi for providing pCMV-YY1, purified YY1, and many helpful discussions. T. Shenk and A. Usheva kindly supplied YY1 antiserum, and J.-M. Mathias provided assistance with in situ hybridization studies. We are grateful for the advice and guidance of many members of the Green lab.

This work was supported by grants from the National Institutes of Health to D.M.M. and M.R.G.

\section{REFERENCES}

1. Adachi, A., H. E. Gendelman, S. Koenig, T. Folks, R. Willey, A. Rabson, and M. A. Martin. 1986. Production of acquired immunodeficiency syndrome-associated retrovirus in human and nonhuman cells transfected with an infectious molecular clone. J. Virol. 59:284-291.

2. Basu, A., K. Park, M. L. Atchison, R. S. Carter, and N. G. Avadhani. 1993. Identification of a transcriptional initiator element in the cytochrome $c$ oxidase subunit $\mathrm{Vb}$ promoter which binds to transcription factors NF-E1 (YY-1, $\delta$ ) and Sp1. J. Biol. Chem. 268:4188-4196.

3. Bauknecht, T., P. Angel, H.-D. Royer, and H. zur Hausen. 1992. Identification of a negative regulatory domain in the human papillomavirus type 18 promoter: interaction with the transcriptional repressor YY1. EMBO J. 11:4607-4617.

4. Clouse, K. A., D. Powell, I. Washington, G. Poli, K. Strebel, W. Farrar, P. Borstad, J. Kovacs, A. S. Fauci, and T. M. Folks. 1989. Monokine regulation of human immunodeficiency virus type 1 expression in a chronically infected human T cell clone. J. Immunol. 142:431-438.

5. Du, H., A. L. Roy, and R. G. Roeder. 1993. Human transcription factor USF stimulates transcription through the initiator elements of the HIV-1 and Ad-ML promoters. EMBO J. 12:501-511.

6. Embertson, J., M. Zupancic, J. Beneke, M. Till, S. Wolinsky, J. L. Ribas, A. Burke, and A. T. Haase. 1993. Analysis of human immunodeficiency virus-infected tissues by amplification and in situ hybridization reveals latent and permissive infections at single-cell resolution. Proc. Natl. Acad. Sci. USA 90:357-361.

7. Embertson, J., M. Zupancic, J. L. Ribas, A. Burke, P. Rasz, K. Tenner-Rasz, and A. T. Haase. 1993. Massive covert infection of helper $\mathrm{T}$ lymphocytes and macrophages during the incubation period of AIDS. Nature (London) 362:359-362.

8. Flanagan, J. R., K. G. Becker, D. L. Ennist, S. L. Gleason, P. H. Driggers, B.-Z. Levi, E. Appella, and K. Ozato. 1992. Cloning of a negative transcription factor that binds to the upstream conserved region of Moloney murine leukemia virus. Mol. Cell. Biol. 12:3844.

9. Folks, T. M., D. Powell, M. Lightfoote, S. Koenig, A. S. Fauci, S. Benn, A. Rabson, D. Daugherty, H. E. Gendelman, M. D. Hoggan, S. Venkatesan, and M. A. Martin. 1986. Biological and biochemical characterization of a cloned Leu- $3^{-}$cell surviving infection with the acquired immunodeficiency syndrome retrovirus. J. Exp. Med. 164:280-290.

10. Garcia, J. A., F. K. Wu, R. Mitsuyasu, and R. B. Gaynor. 1987. Interactions of cellular proteins involved in the transcriptional regulation of human immunodeficiency virus. EMBO J. 6:37613770 .

11. Giacca, M., M. I. Gutierrez, S. Menzo, F. D. de Fagagna, and A.
Falaschi. 1992. A human binding site for transcription factor USF/MLTF mimics the negative regulatory element of human immunodeficiency virus type 1 . Virology 186:133-147.

12. Harrich, D., J. Garcia, F. K. Wu, R. Mitsuyasu, and R. B. Gaynor. 1989. Role of Sp1-binding domains in in vivo transcriptional regulation of the human immunodeficiency virus type 1 long terminal repeat. J. Virol. 63:2585-2591.

13. Jones, K. A. 1989. HIV trans-activation and transcription control mechanisms. New Biol. 1:127-135.

14. Jones, K. A., J. T. Kadonaga, P. A. Luciw, and R. Tjian. 1986 Activation of the AIDS retrovirus promoter by the cellular transcription factor Sp1. Science 232:755-759.

15. Karasuyama, H., N. Tohyama, and T. Tada. 1989. Autocrine growth and tumorigenicity of interleukin 2-dependent helper $T$ cells transfected with IL-2 gene. J. Exp. Med. 169:13-25.

16. Kato, H., M. Horikoshi, and R. G. Roeder. 1991. Repression of HIV-1 transcription by a cellular protein. Science 251:1476-1479.

17. Kim, S., K. Ikeuchi, R. Byrn, J. Groopman, and D. Baltimore. 1989. Lack of a negative influence on viral growth by the nef gene of human immunodeficiency virus type 1. Proc. Natl. Acad. Sci. USA 86:9544-9548.

18. Lee, J.-S., K. M. Galvin, and Y. Shi. 1993. Evidence for physical interaction between the zinc-finger transcription factors YY1 and Sp1. Proc. Natl. Acad. Sci. USA 90:6145-6149.

19. Lui, F., M. A. Thompson, S. Wagner, M. E. Greenberg, and M. R. Green. 1993. Activating transcription factor-1 can mediate $\mathrm{Ca}^{2+}$. and cAMP-inducible transcriptional activation. J. Biol. Chem. 268:6714-6720.

20. Maitra, R. K., N. Ahmad, S. M. Holland, and S. Venkatesan. 1991. Human immunodeficiency virus type 1 (HIV-1) provirus expression and LTR transcription are repressed in NEF-expressing cell lines. Virology 182:522-533.

21. Margolis, D. M., J. M. Ostrove, and S. E. Straus. 1993. HSV-1 activation of HIV-1 transcription augmented by a cellular protein that binds near the initiator element. Virology 192:370-374.

22. Nabel, G., and D. Baltimore. 1988. An inducible transcription factor activates expression of human immunodeficiency virus in $\mathrm{T}$ cells. Nature (London) 326:711-713.

23. O'Shea-Greenfield, A., and S. T. Smale. 1992. Roles of TATA and initiator elements in determining the start site location and direction of RNA polymerase II transcription. J. Biol. Chem. 267:1391-1402.

24. Ostrove, J. O., J. Leonard, K. E. Weck, A. B. Rabson, and H. E. Gendelman. 1987. Activation of the human immunodeficiency virus by herpes simplex virus type 1 . J. Virol. 61:3726-3732.

25. Pantaleo, G., C. Graziosi, J. L. Demarest, L. Butini, M. Montroni, C. H. Fox, J. M. Orenstein, D. P. Kotler, and A. S. Fauci. 1993. HIV infection is active and progressive in lymphoid tissue during the clinically latent stage of disease. Nature (London) 362:355-358.

26. Park, K., and M. L. Atchison. 1991. Isolation of a candidate repressor/activator, NF-E1 (YY1, $\delta$ ), that binds to the immunoglobulin $\kappa 3^{\prime}$ enhancer and the immunoglobulin heavy-chain $\mu \mathrm{E} 1$ site. Proc. Natl. Acad. Sci. USA 88:9804-9808.

27. Seto, E., Y. Shi, and T. Shenk. 1991. YY1 is an initiator sequencebinding protein that directs and activates transcription in vitro. Nature (London) 354:241-245.

28. Shi, Y., E. Seto, L.-S. Chang, and T. Shenk. 1991. Transcriptional repression by YY1, a human GLI-Krüppel-related protein, and relief of repression by adenovirus E1A protein. Cell 67:377-388.

29. Singer, R. H., K. S. Byron, J. B. Lawrence, and J. L. Sullivan. 1989. Detection of HIV-1 infected cells from patients using nonisotopic in situ hybridization. Blood 74:2295-2301.

30. Tiley, L. S., S. J. Madore, M. H. Malim, and B. R. Cullen. 1992. The VP16 transcription activation domain is functional when targeted to a promoter-proximal RNA sequence. Genes Dev. 6:2077-2087.

31. Wu, F. K., J. A. Garcia, R. Mitsuyasu, and R. B. Gaynor. 1988. Alterations in binding characteristics of the human immunodeficiency virus enhancer factor. J. Virol. 62:218-225. 